\title{
Superharp - A Wire Scanner with Absolute Position Readout \\ for Beam Energy Measurement at CEBAF
}

\author{
C. Yan
}

Physics Division, CEBAF

September 7, 1994

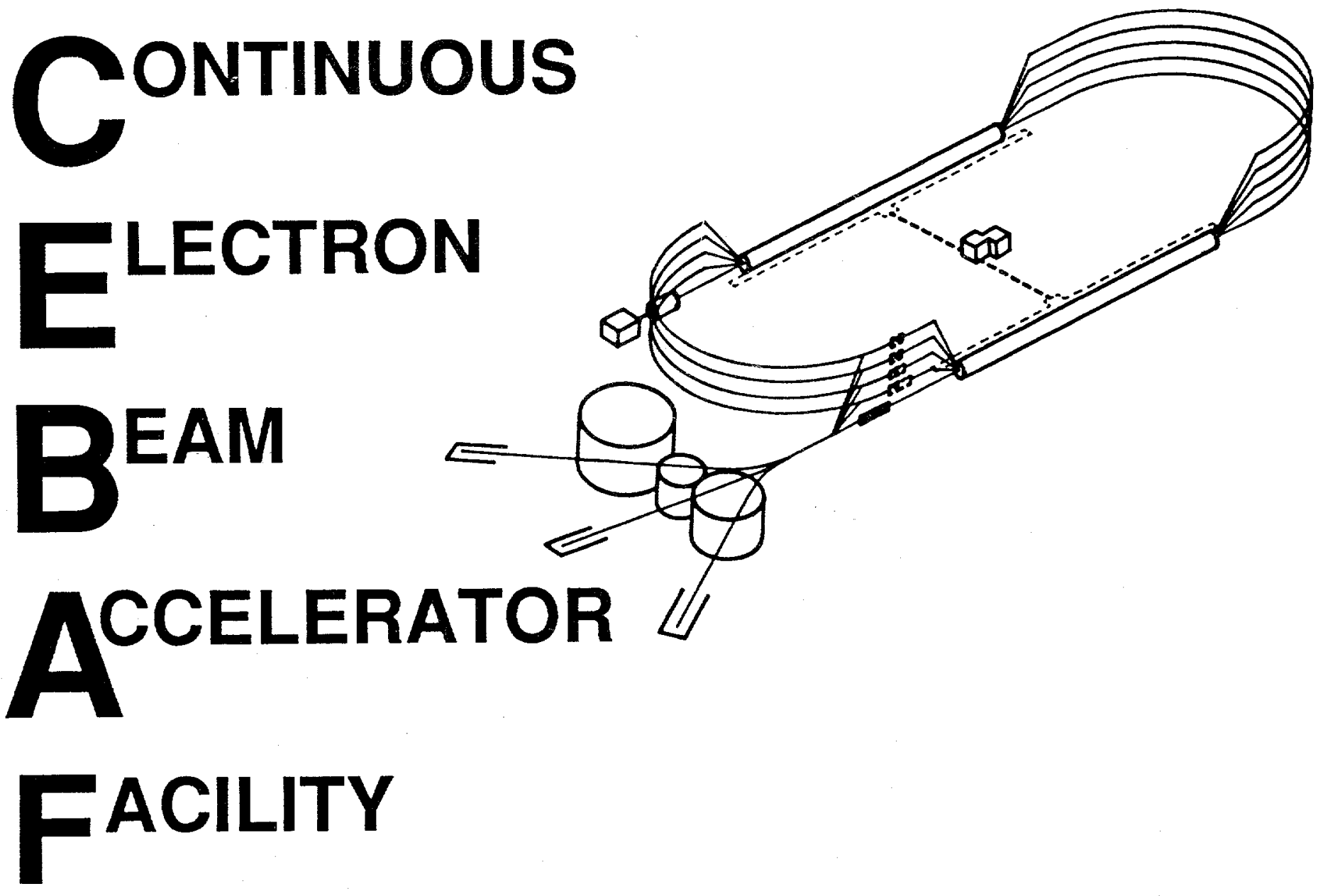

DISTRIBUTION OF THIS DOCUMENT IS UNLIMITED

\section{SURA Southeastern Universities Research Association}

\section{G도운}

The Continuous Electron Beom Accelerator Fachlity

Newport News, Virginia 
Copies available from:

\author{
Library \\ CEBAF \\ 12000 Jefferson Avenue \\ Newport News \\ Virginia 23606
}

The Southeastern Universities Research Association (SURA) operates the Continuous Electron Beam Accelerator Facility for the United States Department of Energy under contract DE-AC05-84ER40150.

\title{
DISCLAIMER
}

This report was prepared as an account of work sponsored by the United States government. Neither the United States nor the United States Department of Energy, nor any of their employees, makes any warranty, express or implied, or assumes any legal liability or responsibility for the accuracy, completeness, or usefulness of any information, apparatus, product, or process disclosed, or represents that its use would not infringe privately owned rights. Reference herein to any specific commercial product, process, or service by trade name, mark, manufacturer, or otherwise, does not necessarily constitute or imply its endorsement, recommendation, or favoring by the United States government or any agency thereof. The views and opinions of authors expressed herein do not necessarily state or reflect those of the United States government or any agency thereof. 


\section{DISCLAIMER}

Portions of this document may be illegible in electronic image products. Images are produced from the best available original document. 


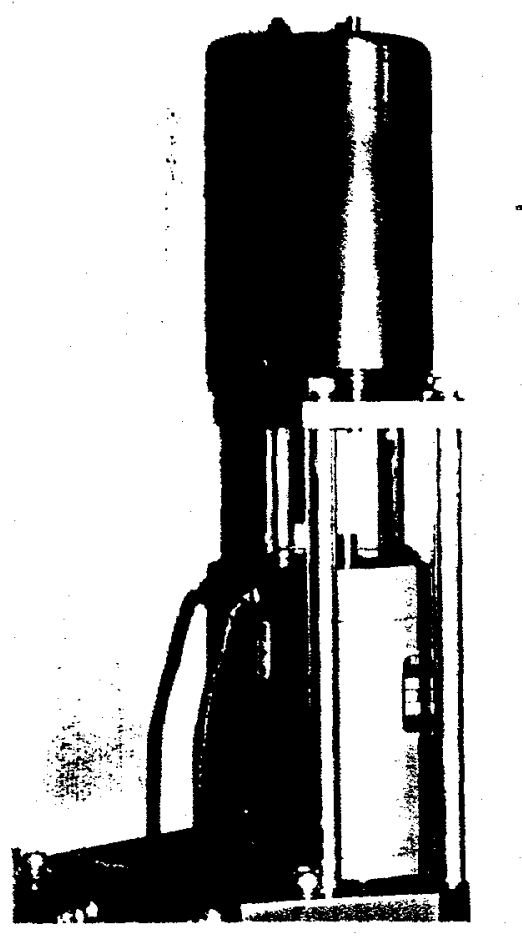

Figure 2: The conjunction part between stepper motor and shaft encoder

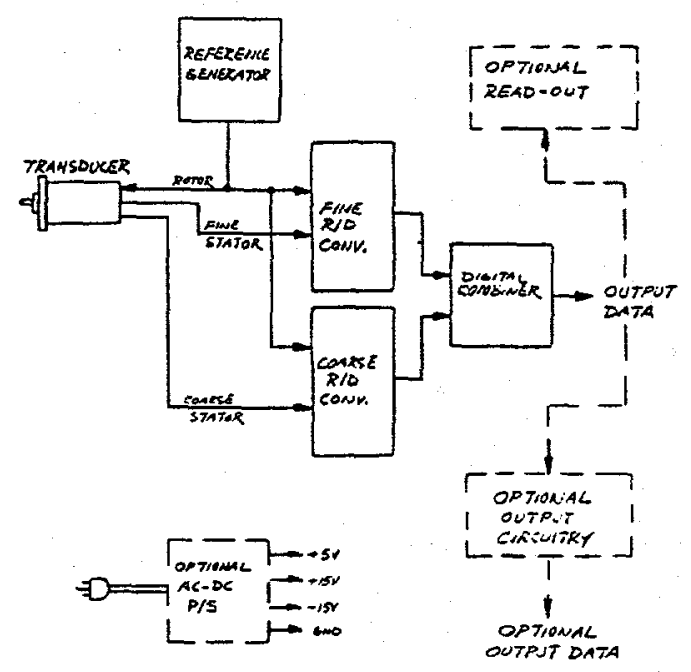

Figure 3: Block diagram of tracking multi-turn shaft resolver
Table 1: Parameters of HTMDS90 encoder

\begin{tabular}{|ll|}
\hline Input & 64 turn NEMA 12 transducer \\
Turns for full counts & 64 \\
Counts per turn & $2^{12}(65536)$ \\
Repeatability & $\pm 1 \mathrm{LSB}$ \\
Accuracy & $\pm 1.526 \times 10^{-5}$ \\
& \pm 1 part in 65536 \\
Angle data output & Parallel binary, 18 bit \\
& resolution, TTL compatible \\
Inhibit/enable & Active low input \\
Converter busy & Positive pulse, 0.5 to 1.5 \\
& $\mu$ s duration \\
Analog output & $\pm 10 \mathrm{~V}$ DC at maximum \\
& tracking rate \\
\hline
\end{tabular}

Table 2: Parameters of superharp motion with 16 bit encoder readout

\begin{tabular}{|ll|}
\hline Pitch of lead screw & $2.54 \mathrm{~mm}$ \\
Distance between & \\
two limit switches & $\sim 3$ inch \\
Microsteps per revolution & 51200 \\
Microsteps per 16 bit count & 25 \\
$\begin{array}{l}\text { Position resolution } \\
\text { (per } 16 \text { bit count) }\end{array}$ & $1.240234 \mu \mathrm{m}$ \\
\hline
\end{tabular}

nal equal to the sine and cosine of the fine and coarse resolvers of the transducer. These signals are then converted to digital by the converters which are fed into the digital combiner which removes the amplitudes and gearing errors.

The Table 1 gives major performance of HTMDS90-64DB18-1P tracking multi-turn encoder.

To match capability of $\mathrm{PC}$ computer, the intermediate 16 bit of 18 bit of the tracking encoder is used to transfer position data and the highest and the lowest bits are eliminated. The basic parameters of superharp moving mechanism are shown in table 2.

16 bit position accuracy is equal to a $1.24 \mu \mathrm{m}$ resolving power of translation motion of shaft encoder, it is good enough for required position measurement precision (better than $10 \mu \mathrm{m}$ ). To avoid from wrap around of 16 bit encoder readout in a 18 bit motion range, the offset (the readout range of 16 bit, which corresponds to the distance between two position limit switches plus harp deacceleration distance after position limit switch is hit) of each encoder has been pre-adjusted to ensure that the reading is between $256-65800$. Encoder tracking unit uses positive logic, the input register uses negative logic. A logic level conversion should be made by software when data signals from encoder tracking unit (positive logic) are transmitted into $60 \mathrm{~A}$ input register (negative logic) to keep a complementary output which indicates the realistic motion.

Because the translation of the wire is generated by the rotation of stepping motor through a rotary axes, the backlash of the transmission screw makes the major contribu- 
tion to the error of the position readout. This will be discussed in error analysis.

\subsection{Improvement of electronics}

The configuration of original preamplifier is based on the isolation amplifier which is used to isolate electrode input and the output for better noise rejection. There is still a severe noise problem caused by switching frequency of stepper motor current driver whenever the harp is moving or stopped and high frequency noise generated by wire - electrode antenna as common mode rejection rate is pretty poor. Sometimes, the profile output is entirely modulated by the noise interference. Even though a fast Fourier trans- formation can be used to separate beam profile from noise background in a time domain [7], but the basic solution should be put on electronics itself.

Low frequency noise is straight forward contributed by switching frequency of the current driver of stepper motor through direct ground coupling between pre-amplifier and stepper motor. Following Nylon insulation couplings are used to connect motor with transmission screw, also motor with encoder. The motor case, the encoder case are separately grounded through a common point.

An AD549 electrometer amplifier with extra high input impedance, extra low offset current is used to replace the isolation amplifier of original CEBAF harp preamplifier. The differential inputs amplifier has very high CMRR (common mode rejection rate) which effectively reduces the high frequency noise picked up by electrode wire through the input ends.

Figure 4 shows a comparison of the noise level between the original isolation amplifier and the new differential input preamplifier during harp when a sine signal is fed to the input of amplifiers. The result noise count is greatly reduced from 363 counts to $20-50$ counts by the procedure mentioned above.

The output of harp preamplifier is then sent to DSP Model 1008 eight channel programmable signal conditioner, which contains eight individual instrumentation amplifiers in a fully programmable CAMAC single width package. The differential input amplifiers with gains up to 1024 are well suited to transmit signal through long cable in a very noisy environments because of good common mode rejection rate up to $100 \mathrm{~dB}$, low noise less than $2 \mu \mathrm{V}$ $V_{p p}$.

\subsection{Study on Vibration Sources}

In order to study wire vibration caused by different sources, microscope video camera is temporarily mounted on the moving plate of superharp as shown in Figure 5. The object lens of camera microscope traces the motion of the wire and the instant wire image is displayed at a fixed position on TV screen. The wire size is used as the reference scale of vibration amplitude. During the wire travels towards and backwards in the whole range, no vibration can be observed, therefore, the moving mechanism (stepper motor, lead screw, and Thomson rods) does not
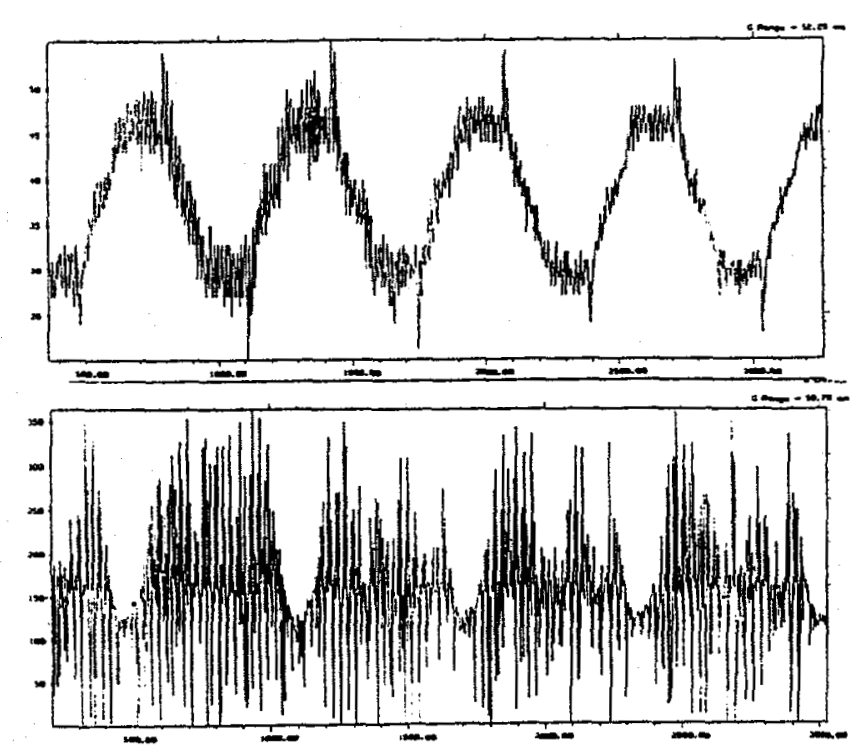

Figure 4: Comparison of noise level of electrometer differential input preamplifier (upper) and the original isolation amplifier (lower)

Table 3: Vertical and horizontal components of ground motion at CEBAF

\begin{tabular}{|llcc|}
\hline Location & Subject & $\mathrm{v}_{v}(\mu \mathrm{m} / \mathrm{s})$ & $\mathrm{v}_{h}(\mu \mathrm{m} / \mathrm{s})$ \\
EEL 122 & $\begin{array}{l}10^{\prime} \times 4^{\prime} \text { 'steel } \\
\text { optical bench }\end{array}$ & 3.5 & 7 \\
EEL 126 & $\begin{array}{l}6^{\prime} \times 4^{\prime} \text { granite } \\
\text { table with full load }\end{array}$ & 10.0 & 14 \\
EEL Hall & $\begin{array}{l}8^{\prime} \times 4^{\prime} \text { granite } \\
\text { table without load } \\
\text { QD0L09 girder }\end{array}$ & 11 & 7 \\
Injector & 14 & 17.8 \\
\hline
\end{tabular}

cause wire vibration. The wire vibration with amplitude larger than $10 \mu \mathrm{m}$ is generated by ground motion and adjacent mechanical noise sources (for example, the cooling water running through the pipe, vibration of mechanical pumps).

To achieve a high accuracy of beam position measurement, wire vibration must be suppressed not only by optimizing the harp configuration but also effectively damping all possible vibrations. One major reason that causes wire vibration is mechanical motion of ground by local traffic and night coal train.

A pair of accelerometers (L-10, horizontal sensor and vertical sensor, Martin products) are used to find out local ground motion amplitude at different locations. The calibrated voltage output of accelerometers is $0.7 \mathrm{~V} /$ (inch/second) on a $75 \Omega$ terminator. Table 3 gives the measuring results. 


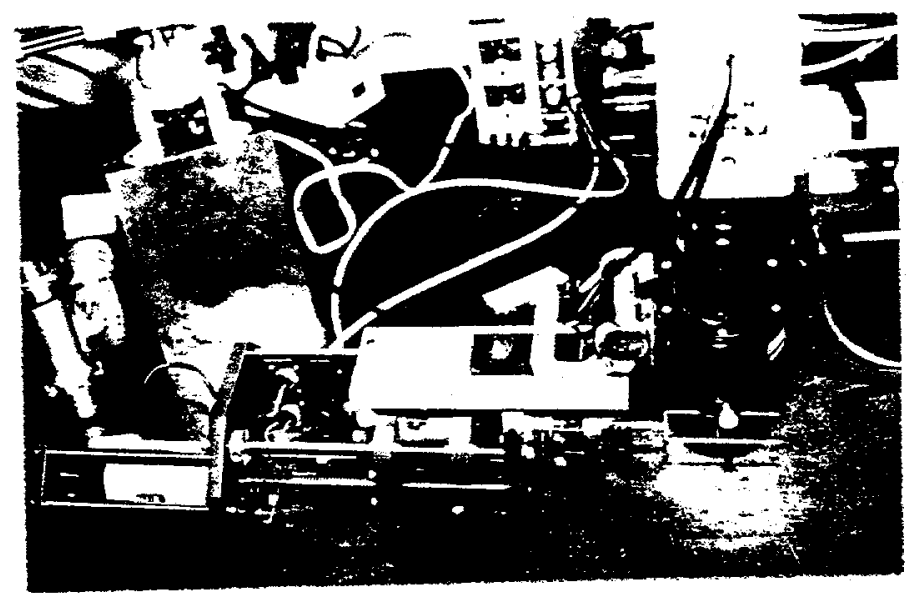

Figure 5: The microscope video camera system mounted on the harp driving plate tracing the sensor wire in the whole motion range for test of instant wire vibration caused by moving mechanism

\subsection{Improvement of Support Mechanism}

In order to damp vibration from all kind of sources, instead of beam line aluminum girder, a heavy weight granite table is installed as the foundation of superharp support mechanism. At the same location, for example, at the position of second pair of superharps (IHA3C12A, IHA3C12B), the ground vibration amplitude can be reduced by factor 5 after granite table is installed. The slight mechanical vibration caused by other sources is also effectively absorbed by granite table. On the granite table superharps are decoupled with other beamline elements by bellows.

\subsection{Mechanical Configuration of the fork}

A further improvement was made on the fork mechanical configuration. A balsa fork is used to replace original aluminum one because its resonance frequency is much higher than the one of aluminum fork. The weight of balsa fork is about $1.7 \mathrm{~g}$, it is lighter than aluminum one. The balsa fork is also more rigid than the aluminum one because of its anisotropic fiber structure. During wire assembly procedure the balsa fork is clamped in an aluminum fixture with dowel pins on it. The wire orientation is defined by the dowel pins, on which the wire is wrapped around. The tail of the wire is led over the radiuses edge of the fixture and weighted creating a constant tension keeping the wire taut during scans when the wire is significantly heated by beam. The wire is finally glued on the balsa arm by TorrSeal.

This special wire installation mechanism is illustrated in Figure 6

Balsa forks are pre-vacuumed in a bell jar by a rough pump. After the sensor wire is mounted, the fork is kept in the bell jar to avoid humidity from environment. No gas release problem was found for a thoroughly dried, carefully stored harp fork during prototype beam testing experiment in December 1993 and Hall C arc beam line oper-

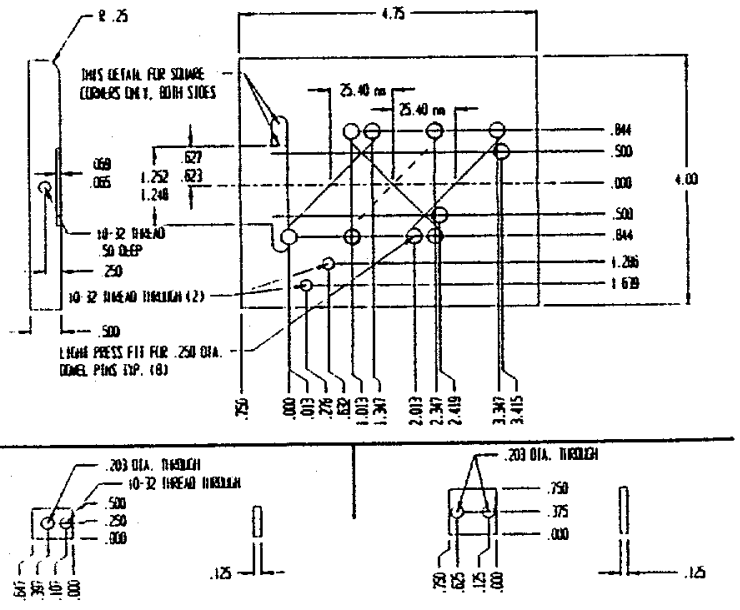

Figure 6: Aluminum fixture and balsa fork assembly

ation in July 1994. During survey process the fork stayed in the air for two weeks, no distortion of balsa fork was found.

The wire orientation is verified and adjusted on optical comparator (Jones and Lawson, Coordinate Measuring Machine) as shown in Figure 7.

On the optical comparator the fork image is enlarged by a magnification of 10 and is displayed on the goniometric optical screen. The angular accuracy of wire orientation is determined by comparator within $\pm 0.1^{\circ}$ at $45^{\circ}$ tilt angle.

\subsection{Survey on superharp}

The geometric center of the cross referring to an external fiducial (tooling ball), which is temporarily installed on the flange of the standard cross, can be found by survey. In the survey procedure, the harp is moved to the position of geometric center of the cross (beam pipe), the corresponding encoder readout is defined as "zero" position of the cross coordinate. The "zero" position is defined as coordinate reference for beam energy determination, the beam positions at different locations of arc spectrometer are measured in this cross coordinate system. After surveying on each harp's own cross, two superharps are installed on the granite table as shown in Figure 8. The alignment is then performed between the two superharps. The harp fiducialization error is estimated as $50 \mu \mathrm{m}$ ( $95 \%$ confidence level, $\sim 4 \sigma$ ), harp to harp survey error about $100 \mu \mathrm{m}$ [8]. There is a slight difference between survey errors in the air and in the vacuum because backlash of the transmission screw will disappear in vacuum under the single direction force generated by outside air pressure. 


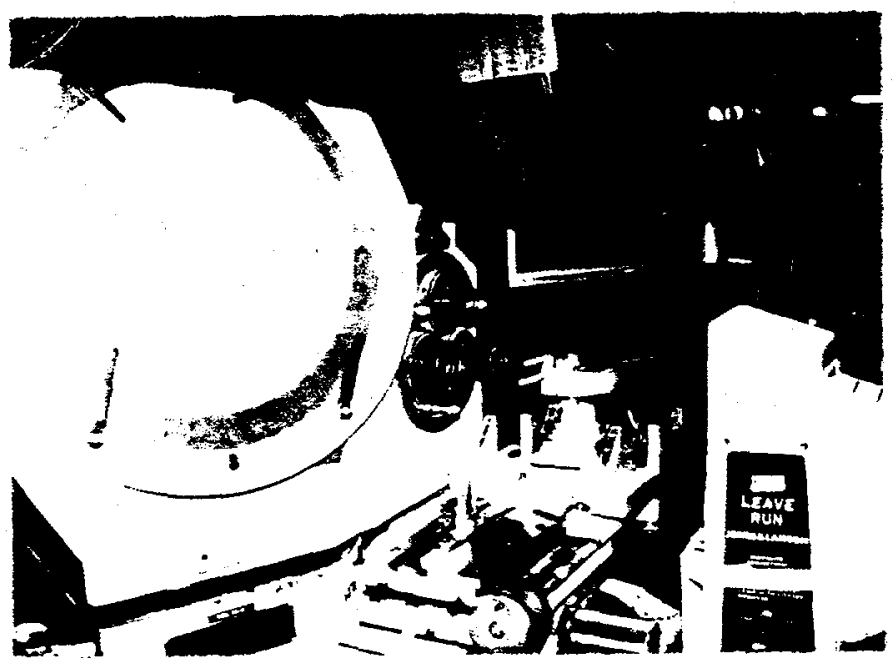

Figure 7: The wire orientation on balsa fork is checked on the optical comparator

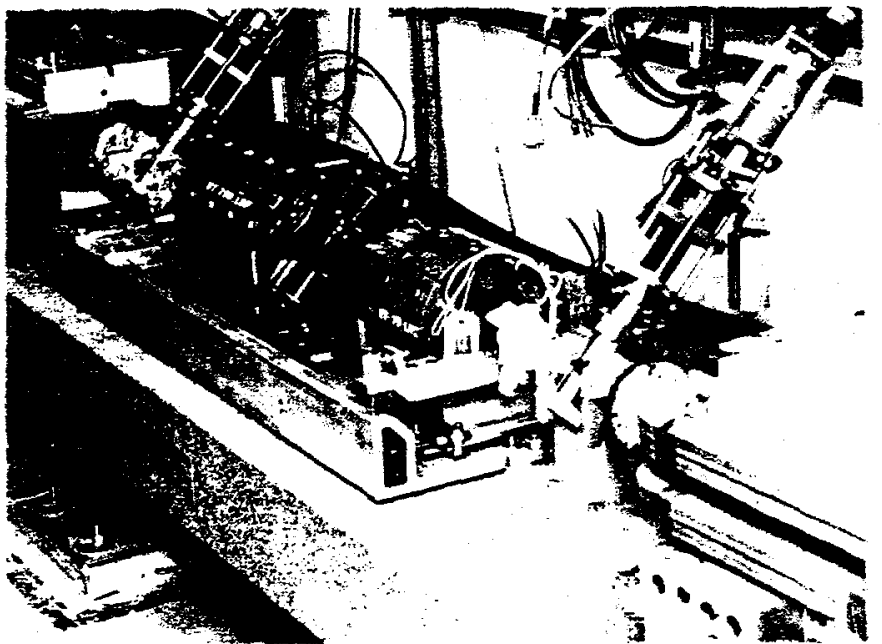

Figure 8: Two superharps are installed on granite table

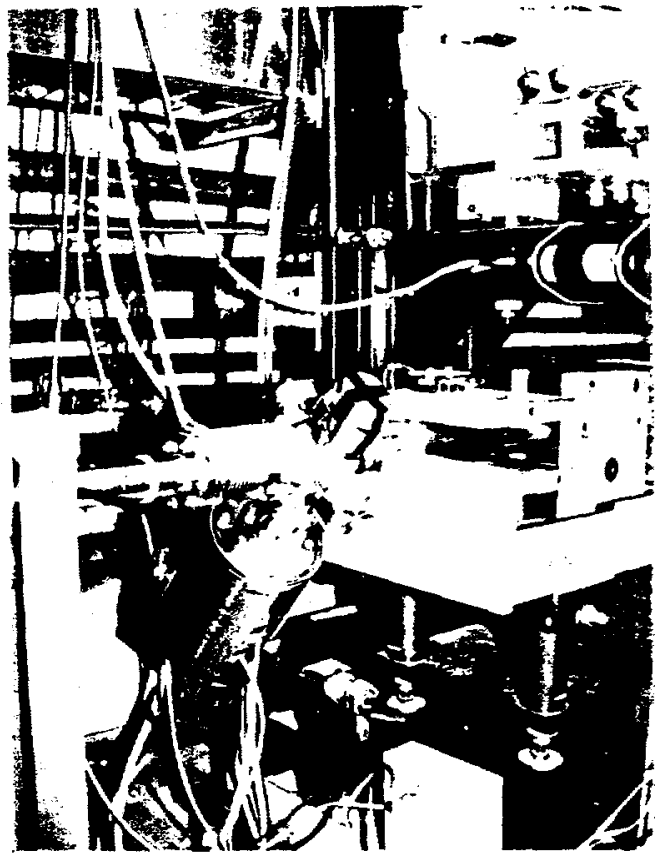

Figure 9: Superharp prototype is installed at the edge of the girder 0L09. The beam loss monitor is located above the $\mathrm{x}-\mathrm{y}$ beam steering magnets on the girder

\section{EXPERIMENTAL TEST OF SUPERHARP PROTOTYPE}

The purpose of the first experimental test is to: a) verify normal operation of superharp prototype with absolute position encoder as the position sensor; $b$ ) improve current sensitivity and broaden current dynamic range from $1 \mu \mathrm{A}$ to $100 \mu \mathrm{A} ; \mathrm{c}$ ) improve signal/noise ratio. In order to study b) and c), two analog signal pick-up channels - direct current measurement by wire electrode and bremsstrahlung detection are independently used to determine beam profile (current profile and radiation profile).

\subsection{Experimental Apparatus}

The superharp prototype was located on the girder OL09 at CEBAF injector section as shown in Figure 9.

Three parallel tungsten wires with different diameters $(10 \mu \mathrm{m}, 22 \mu \mathrm{m}$, and $50 \mu \mathrm{m})$ were mounted on the balsa fork for observation of vertical beam profiles with different current sensitivity. A beam loss monitor was installed 1 meter downstream the superharp for radiation profile measurement. A beam viewer was installed on the girder OL08, $20 \mathrm{ft}$ upstream from superharp and a regular CEBAF harp was settled on girder OL10, $20 \mathrm{ft}$ downstream.

The PC based superharp prototype data requisition system was located in north linac service building. A block diagram of electronics used in the tests is shown in Figure 10.

The beam energy at CEBAF injector is $45 \mathrm{MeV}$, duty factor $7.5 \times 10^{-3}$, the pulse width is $125 \mu \mathrm{s}$, and frequency is $60 \mathrm{~Hz}$. During the experiment peak beam current was tuned from $1 \mu \mathrm{A}$ to $115 \mu \mathrm{A}$. 


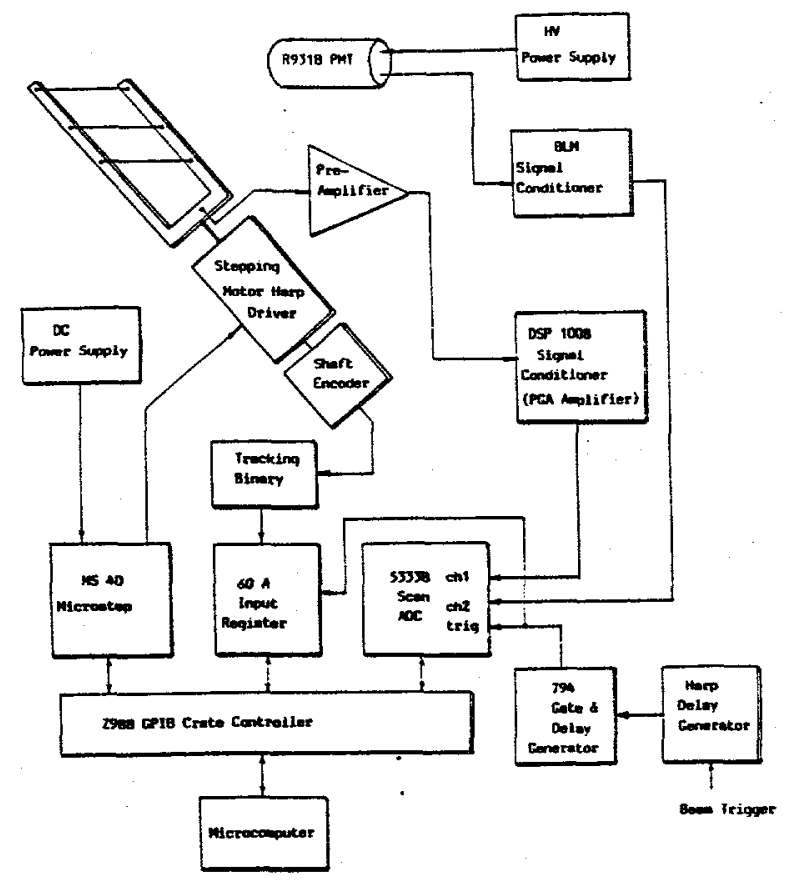

Figure 10: Block diagram of superharp prototype electronics

Table 4: Major specification of Hamamatsu 931B side-on photomultiplier

\begin{tabular}{|ll|}
\hline Spectrum range & $300-660 \mathrm{~nm}$ \\
Cathode luminosity response & $40 \mu \mathrm{A}$ per $\mathrm{Im}$ \\
Current gain & $6 \times 10^{6}$ \\
Rise time & $1.8 \mathrm{~ns}$ \\
Transit time & $18 \mathrm{~ns}$ \\
Number of dynodes & 9 \\
\hline
\end{tabular}

\subsection{Radiation Profile Pick-Up}

The radiation level near the sensor wire which is moving across the electron beam will be entirely dominated by the bremsstrahlung $\mathrm{X}$ or gamma rays produced in the target wire. This local dose rate will be a function of emission angle and also be dependent on wire diameter and the presence of surrounding material. The dose rate at BLM location ( $1 \mathrm{~m}$ from sensor wire with $30^{\circ}$ emission angle) for per $\mathrm{kW}$ beam power loss can be estimated by the formula [9]:

$$
D=2700 \sqrt{E} \theta^{-1.5} G y h^{-1} k W^{-1}
$$

where $\mathrm{E}$ is the electron energy in $\mathrm{MeV}$ and $\theta$ the emission angle in degree. When $45 \mathrm{MeV} 1 \mu \mathrm{A}$ electron beam is scanned by the sensor wire, the beam power loss in the wire is about $1.08 \mu \mathrm{W}[6]$ and corresponding dose rate is approximately $112 \mu \mathrm{rad}$ per hour that is the dose detected by the BLM channel of superharp system when the wire is crossing the beam.

The radiation detector is a Hamamatsu 931B side-on photomultiplier with general specification listed in Table 4.

No scintillator is required for Hamamatsu $931 \mathrm{~B}$ photomultiplier. The side-on glass envelope generates

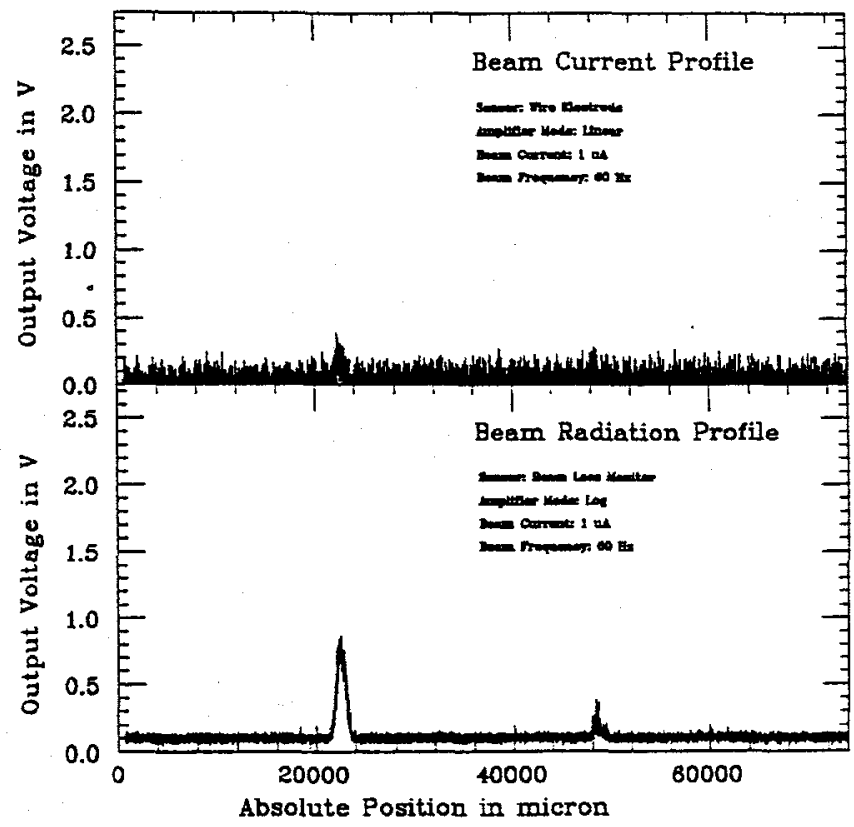

Figure 11: Current profile (upper) and Bremsstrahlung profile (lower) for $45 \mathrm{MeV} 1 \mu \mathrm{A}$ beam

scintillation and $\hat{C}$ herenkov radiation under a certain Bremsstrahlung radiation environment when energetic electron beam bombarding the wire. When electron beam interacts with wire, the primary radiation - Bremsstrahlung $\mathrm{x}$-ray is produced. With increasing the energy of electrons the following secondary emissions take into place: electron-positron pair (electromagnetic shower); photonuclear interaction - photo-neutrons; neutron-proton pair; photo pion; and muon pair. When CEBAF electron beam energy increases from $45 \mathrm{MeV}$ to 4 $\mathrm{GeV}$, the dose rate will increase by factor 3 with stronger forward angle emission. This is why one beam loss monitor can be used to observe radiation beam profiles from two cascade superharps on each granite table while those two harps are operated independently.

The signal conditioner of BLM is a separate card within the BLM controller module. Either linear or logarithmic signal conditioning mode can be used for different functions. In the case of superharp, BLM conditioner is switched to logarithmic mode which covers very wide dynamic range (11 decade). The logarithmic mode is specially used to extract low level signals from noise background. For either two conditioning mode, the input signal range is converted to a $0-5$ volt voltage range for the data acquisition network.

\subsection{Experimental Beam Profiles}

A series of beam profiles were obtained during the superharp prototype on beam testing at different beam current from $1 \mu \mathrm{A}$ to $125 \mu \mathrm{A}$. Each profile plot consists of current profile and Bremsstrahlung profile in the same position coordinate. Two peaks, corresponding to $10 \mu \mathrm{m}$ and $22 \mu \mathrm{m}$ wire respectively, are displayed in beam profile plot and the third $50 \mu \mathrm{m}$ wire is out of motion range.

Figure 11 shows the worse beam profile with a beam 


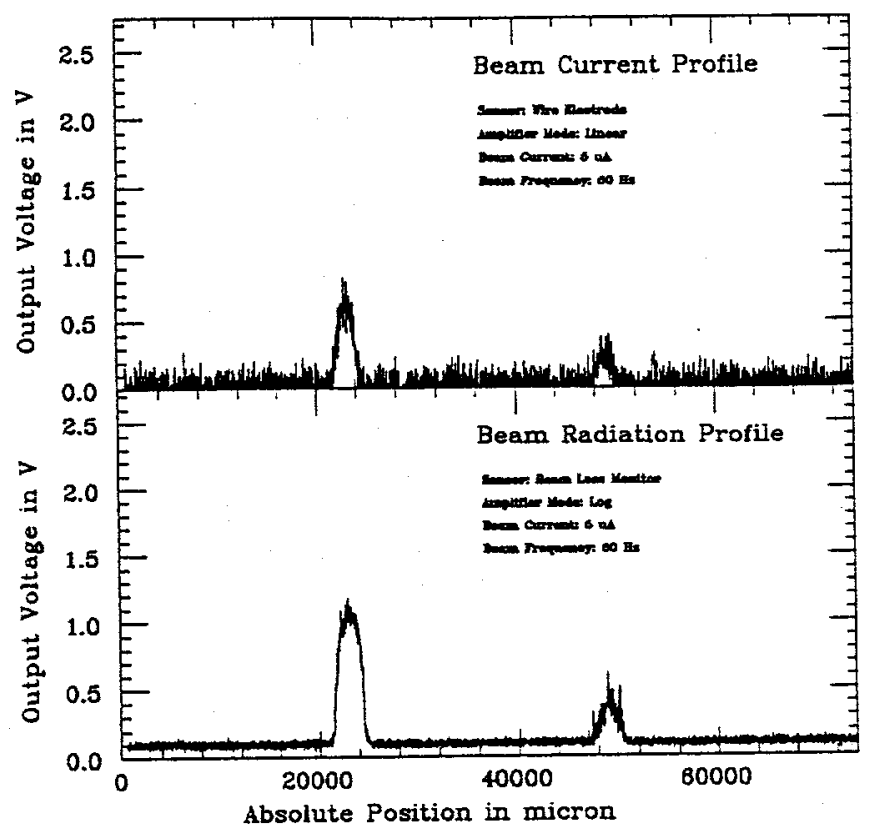

Figure 12: Current profile (upper) and Bremsstrahlung profile (lower) for $45 \mathrm{MeV} 5 \mu \mathrm{A}$ beam

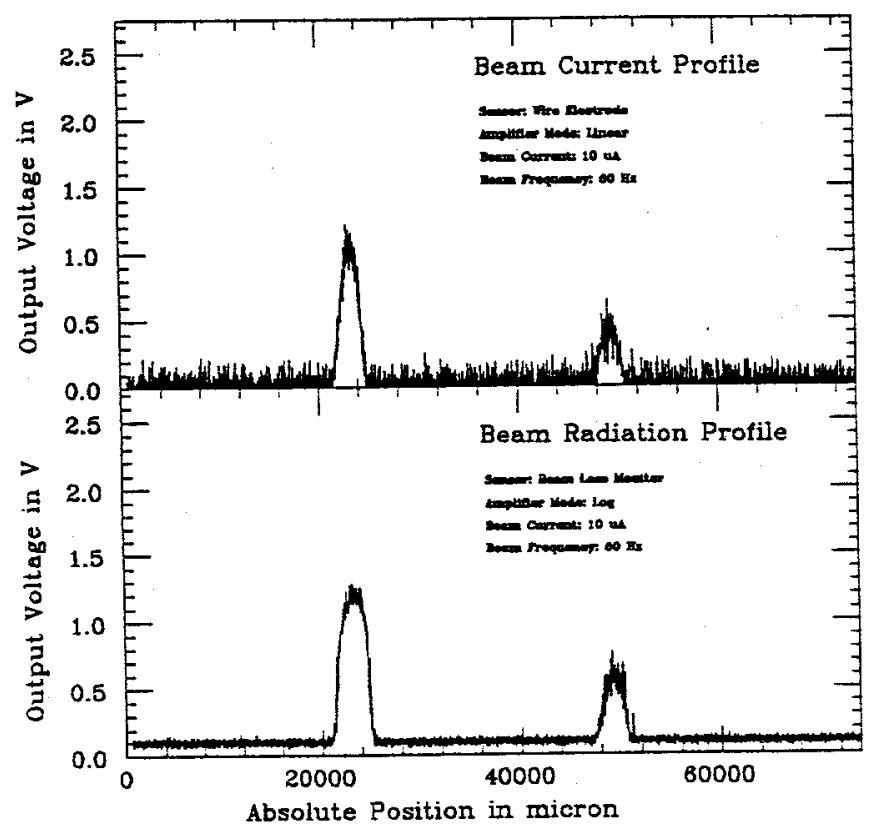

Figure 13: Current profile (upper) and Bremsstrahlung profile (lower) for $45 \mathrm{MeV} 10 \mu \mathrm{A}$ beam

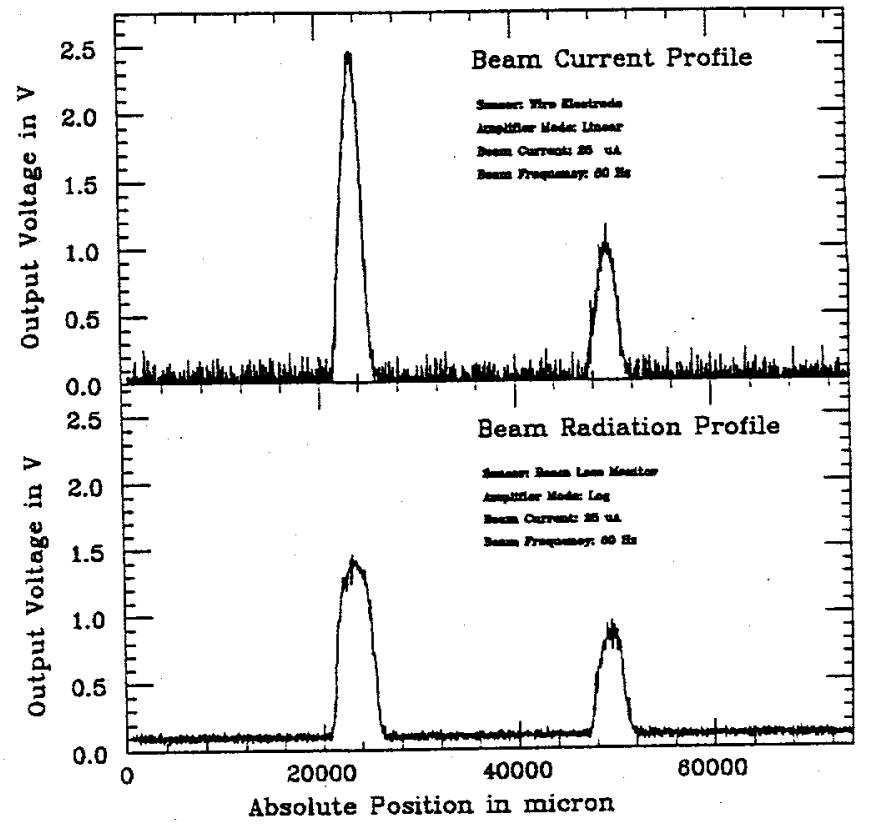

Figure 14: Current profile (upper) and Bremsstrahlung profile (lower) for $45 \mathrm{MeV} 25 \mu \mathrm{A}$ beam

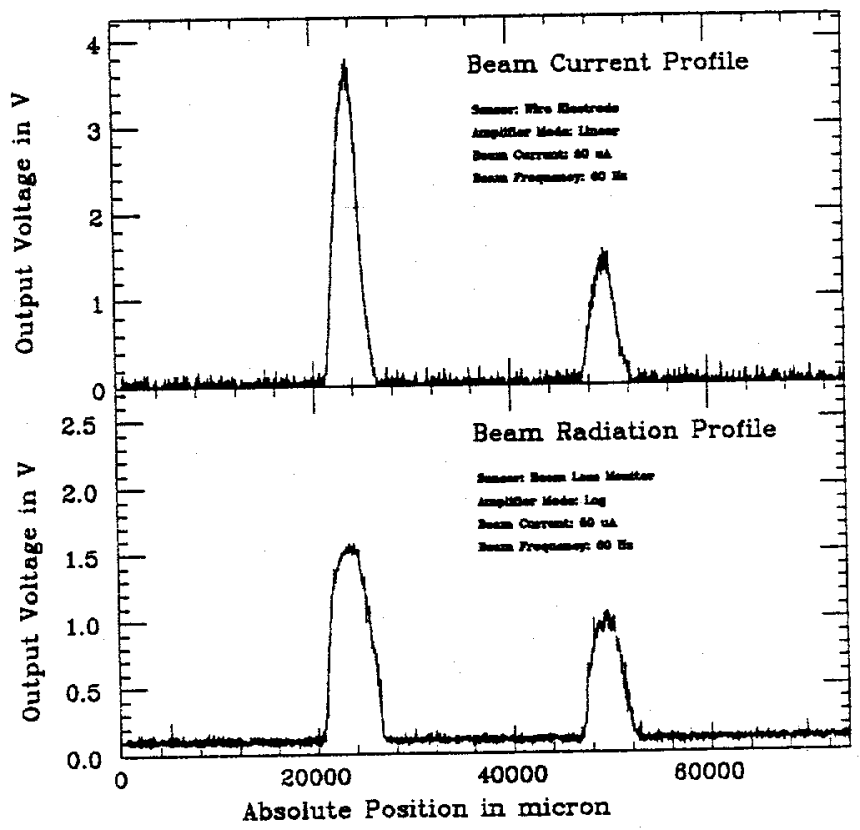

Figure 15: Current profile (upper) and Bremsstrahlung profile (lower) for $45 \mathrm{MeV} 50 \mu \mathrm{A}$ beam 


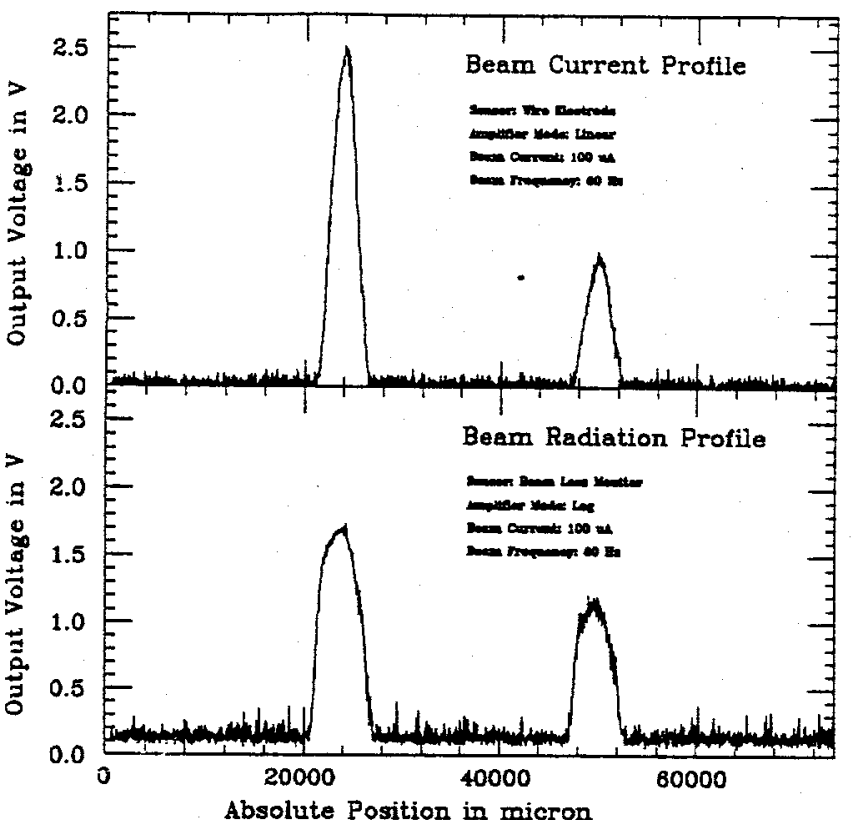

Figure 16: Current profile (upper) and Bremsstrahlung profile (lower) for $45 \mathrm{MeV} 100 \mu \mathrm{A}$ beam

Table 5: Comparison between current profile and Bremsstrahlung profile at $1 \mu \mathrm{A}$ beam current

\begin{tabular}{|lll|}
\hline & Current & Bremsstrahlung \\
Average noise $(\mathrm{V})$ & 0.034 & 0.05 \\
Peak noise & 0.27 & 0.14 \\
10 $\mu$ m wire peak & 0.28 & 0.44 \\
S/N ratio $(10 \mu \mathrm{m})$ & 1.04 & 3.14 \\
$22 \mu \mathrm{m}$ wire peak & 0.39 & 0.92 \\
S/N ratio $(22 \mu \mathrm{m})$ & 1.44 & 6.57 \\
\hline
\end{tabular}

current of $1 \mu \mathrm{A}$. In upper current profile the average $\mathrm{S} / \mathrm{N}$ ratio is about 1.04 at $10 \mu \mathrm{m}$ wire, 1.44 at $22 \mu \mathrm{m}$ wire, and this was greatly improved by Bremsstrahlung logarithmic system. A N/S ratio comparison between current profile and radiation profile at $1 \mu \mathrm{A}$ current (motor speed: 2000 steps/second, the gain of programmable amplifier: 64) is shown in Table 5.

From experimental data mentioned above, several technical highlights could be summarized as the following:

- Bremsstrahlung beam profile has higher sensitivity than current pick-up at low current, specially below 5 $\mu \mathrm{A}(45 \mathrm{MeV})$.

- One beam loss monitor can be used as common Bremsstrahlung profile pick-up sensor for two cascade superharps when beam energy increases to $\mathrm{GeV}$ because the enhanced forward angle radiation

- To prevent saturation effect of beam loss monitor, the distance between BLM location and superharp can be increased to $200-300 \mathrm{~cm}$, also the emission angle could be set beyond 30 degree at higher beam energy because dose yield is increased.

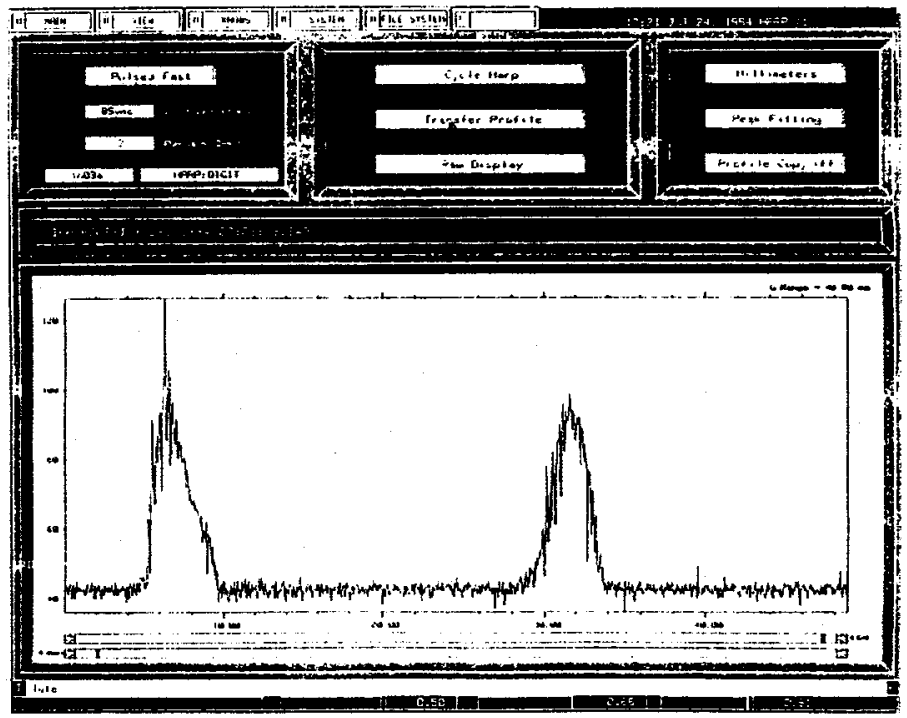

Figure 17: x-y current profiles for $600 \mathrm{MeV} 25 \mu \mathrm{A}$ pulsed beam

- The linear current channel provides a non-distorted beam profile, which has higher accuracy to determine the center of mass of beam profile as "beam position". The adequate range is from $5 \mu \mathrm{A}$ to $100 \mu \mathrm{A}$.

- $22 \mu \mathrm{m}$ diameter tungsten wire was selected as sensor wire because of its good balance between sensitivity and position resolution.

\section{SUPERHARPS INSTALLATION}

Based on the successful experiment result of superharp prototype beam testing in December 1993, eight superharps were installed on Hall C beam line in July 1994. Six of them were installed in BSY arc section for beam energy measurement in nearly future, the final two were installed in chicane section just in front of target as regular beam profile monitors. The first $600 \mathrm{MeV}$ CEBAF electron beam profile was obtained during this run as shown in Figure 17.

Two $22 \mu \mathrm{m}$ wires which are perpendicular each other were installed on the fork. When the harp scans with $45^{\circ}$ tilt angle, the beam horizontal profile and the vertical one are displayed successively. The profile on the left side is vertical and the horizontal one is on the right side.

A 17-th bit cable was added on the 16 bit cable between encoder interface (tracking unit) and the Jordan input register in order to keep data effective in any case.

Some special noise problems occured during the installation of eight superharps on Hall $\mathrm{C}$ beam line and they were greatly suppressed by systematic study on the local electric connection and grounding path.

The cross talk between preamplifier and reference frequency generator of the encoder was entirely suppressed by firmly connecting encoder enclosure and preamplifier box. 


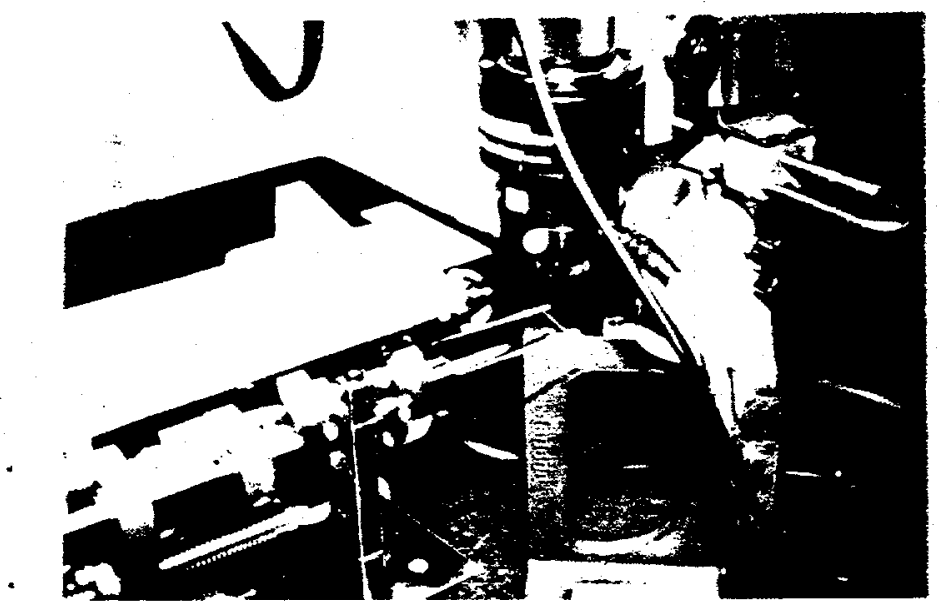

Figure 18: The video microscope camera system installed on optical bench looking at the wire position before and after scan to determine wire motion repeatability

The $60 \mathrm{~Hz}$ spike type noise with few volt amplitude comes from different grounding level between beam line and superharp system and improper grounding of superharp electronic system. Reconnecting common ground cable of beam line to preamplifier box made spike noise disappeared.

Standing waveform "Ring" noise is caused by mechanical vibration at partial travel of the harp scan (mostly near one end of moving range) while a misalignment of Thomson rods exists. Realignment of Thomson rods should be done until getting vibration vanished.

\section{POSITION ERROR ANALYSIS}

In order to test the repeatability of the motion of the superharp, a microscope video camera is mounted on a fixed stands on the granite table as shown in Figure 18. By looking at encoder readout, the harp is driven through entire motion range ( 3 inch) and then back to the original position. The position repeatability includes position error caused by backlash of driving screw, the error of encoder readout, and position uncertainty of wire size. The repeatability of wire motion is experimentally found to be better than $10 \mu \mathrm{m}$. In survey process, under observation by the instrumentation telescope, the wire motion repeatability is about $20 \mu \mathrm{m}$. The two different data comes from either different precision of measuring systems (Microscope and telescope) or different accuracy of transmission screws adjustment on a certain harp.

For beam energy measurement the absolute beam position is obtained from beam profile data by superharp system. The harp absolute position accuracy is required to be better than $50 \mu \mathrm{m}$, which is equivalent to $3 \times 10^{-5}$ contribution to the energy error. There are several factors which influent wire position accuracy beside survey error.

The smaller the wire size, the better the position resolution in the sense of differential current measurement during a complete scan. In fact, wire size is determined by
Table 6: Error Sources

\begin{tabular}{|lc|}
\hline Classified error source & Figures $(\mu \mathrm{m})$ \\
Mechanical error: & \\
Uncertainty due to wire size & 2 \\
Backlash of transmission screw & 10 \\
Encoder resolution & 1.24 \\
Wire vibration & 2 \\
Body deformation (long term) & 5 \\
Wire deformation (long term) & 5 \\
Angular misalignment of fork & 4 \\
Subtotal (Repeatability) & 13 \\
Survey error: & \\
Harp fiducialization & \\
error & 50 \\
\hline
\end{tabular}

both beam size and the requirement of minimum current sensitivity. For $100 \mu \mathrm{m}$ beam size the wire size contribution to position uncertainty is not sensitive and $22 \mu \mathrm{m}$ wire is chosen for superharp.

Backlash of transmission screw is a major contribution to possition error. The backlash is estimated not larger than $10 \mu \mathrm{m}$ for $2.54 \mathrm{~mm}$ pitch lead screw. In vacuum the nut plate experiences a constant force only in one direction and the backlash entirely vanishes.

The sensor wire becomes loose when there is certain deformation of balsa fork. All balsa material and forks were pre-vacuumed and stored in a bell jar. After calibration and installation no fork deformation can be observed. Due to there is a pre-tension as the wire is installed on the fork, the wire deformation within a long term is restrivted less than $5 \mu \mathrm{m}$.

Classified error sources are listed on Table 6

\section{EVALUATION ON PRESENT SYSTEM}

- The basic performance of present superharp system meets the requirement of $10^{-3}$ beam energy measurement.

- Stepper motor driving system can be simplified by using various translation driving mechanism because the instant position readout is only determined by encoder.

- To avoid from coupling problem and to reduce position error, the best way to measure transverse displacement of the wire of scanner is to use linear encoder firmly connected with the moving part of the harp. The common used optical linear encoder could reach the order of submicron within $1000 \mathrm{~mm}$ range (Heidenhain). With linear encoders featuring distance-coded reference marks, the absolute position value can be re-established by crossing over two consecutive reference marks (after a traverse of $20 \mathrm{~mm}$ ). 
The major elements of incremental linear encoder are high density optical grating and the photoelectric readout system. Careful study on local radiation level in BSY arc beam line is necessary.

- Beam loss monitor is suitable for the use of the only analog sensor on superharp and current pick up channel can be eliminated because the BLM conditioner can be operating in either logarithmic mode or linear mode and Hamamatsu 931B tube can also be replaced by 12 dynodes $30 \mathrm{~mm}$ diameter FEU-115M PM. It will have higher sensitivity and wider dynamic range.

- The wire holding system and wire survey procedure has to be simplified to meet the requirement of fast wire replacement in a 1-2 year period. (from SLC experience, about $90 \%$ of the $40 \mu \mathrm{m}$ diameter wires have broken during routing use.) A technique to realize highly reproducible wire-fork installation is to be developed.

- To meet the procedure of beam energy measurement, EPICS based software needs a further development to provide dual beam profile measurement and display, high precision beam centroid algebra, and automatic control for sequential scan of 6 superharps in arc spectrometer.

\section{ACKNOWLEDGEMENTS}

The CEBAF superharp wire scanners have performed effectively, providing the tools needs to carry out beam energy measurement and beam profile measurement in BSY arc section for either physics experiments or BSY beam line optical tuning. This is due to the collective efforts of CEBAF engineers, technicians, designers and physicists. Special mention should be made of the following individuals who have been involved in different activities.
Activity

Project initiation

Coordinator

Mechanical design

Bench testing of

superharp prototype

Mechanical assembly

PC based control system

Balsa harp mechanism

Preamplifier

Prototype beam testing

Design of granite table

and beam line support

Installation on beam line

Survey and calibration

Beam line electronics

installation

System verification

EPICS based software

Software development

System development

Documentation
Persons involved

C. Yan

R. Carlini, J. Denard

E. Feldl -

C. Yan, H. Feng

P. Adderley, E. Feldl

P. Adderley

R. Wojcik

B. Kross, J. Van Dyke

K. Jordan

R. Wojcik, C. Yan

J. Van Dyke, H. Fanning

S. Williams, B. Kross

J. Dahlberg

K. Capek, A. Silva

J. Beaufait

K. Capek, A. Silva

D. Barker, C. Yan

D. Barker, B. Bruce

D. Barker, S. Wood

J. Van Dyke, B. Kross

C. Yan

C. Yan

\section{REFERENCES}

[1] C. Yan, R. Carlini, J. Napolitano, D. Neuffer CEBAF-R92-003, March 25, 1992

[2] D. Neuffer, C. Yan, R. Carlini CEBAF-TN-92-054, November 24, 1992

[3] R. V. Servranckx CEBAF-TN-92-044, September 29, 1992

[4] C. Yan, R. Carlini, D. Neuffer CEBAF-PR-93-004, Also Proceedings of the 1993 Particle Accelerator Conference, p. 2136, Volume 3 of 5, May 1993, Washington D.C.

[5] C. Yan, P. Adderley, R. Carlini, E. Feldl, H. Feng CEBAFTN-93-006, January 16, 1993

[6] C. Yan, CEBAF-TN-1993-014 January 20, 1994

[7] R. Li, CEBAF-TN-1993-010, January, 1993

[8] W. Oren, Minutes of Meeting on beam lines to experimental areas, CEBAF, October 15 and 29, 1992.

[9] A. H. Sullivan "A guide to radiation and radioactivity levels near high energy particle accelerator", p.76, Nuclear Technology Publishing, England, 1992 